\title{
Drug-release system of microchannel transport used in minimally invasive surgery for hemostasis
}

This article was published in the following Dove Medical Press journal:

Drug Design, Development and Therapy

\section{Hong Huang ${ }^{1, *}$ \\ Houhe Liu',* \\ Hua Zhou ${ }^{1, *}$ \\ Zhiling Liang ${ }^{2}$ \\ Dandan Song ${ }^{2}$ \\ Yun Zhang ${ }^{2}$ \\ Wanqiu Huang ${ }^{2}$ \\ Xiaotian Zhao ${ }^{2}$ \\ Bo $\mathrm{Wu}^{2}$ \\ Guodong $\mathrm{Ye}^{2}$ \\ Yugang Huang²}

'Key Laboratory for Major Obstetric Diseases of Guangdong Province, Department of Pharmacy, The Third Affiliated Hospital of Guangzhou Medical University, Guangzhou 510150, China; ${ }^{2}$ Key Laboratory of Molecular Target and Clinical Pharmacology of Guangdong Province, School of Pharmaceutical Sciences, The Fifth Affiliated Hospital of Guangzhou Medical University,

Guangzhou 5I I436, China

*These authors contributed equally to this work
Correspondence: Guodong Ye; Yugang Huang

Key Laboratory of Molecular Target and Clinical Pharmacology of Guangdong Province, School of Pharmaceutical Sciences, The Fifth Affiliated Hospital of Guangzhou Medical University, No 62I, Gangwan Road, Huangpu District,

Guangzhou 511436, China

Tel +86 I32 29494539

Email gzhygd@gzhmu.edu.cn; hyug@gzhmu.edu.cn

\begin{abstract}
Background: Sucrose allyl ether (SAE) containing hemostatic drugs and a photoinitiator was established to treat mild postpartum hemorrhage or long-term continuous abnormal uterine bleeding in minimally invasive surgery (MIS) using a photopolymerization method.

Methods and results: Real-time infrared spectroscopy and rheological experiments showed that the SAE monomer with shear-thinning characteristics could polymerize rapidly into a transparent membrane. Cytotoxicity experiments in vitro showed that this system could elicit a long-term hemostatic effect. Tissue adhesion was also evaluated. The photo-stability of four delivered antifibrinolytic drugs (6-aminocaproic acid, ethylenediaminediacetic acid, tranexamic acid and p-(aminomethyl) benzoic acid) was tested by ultraviolet-photolysis experiments and illustrated by time-dependent density functional theory. Sustained-release experiments revealed that the formed film could be used as a drug carrier. Molecular docking and molecular dynamics were done to investigate the binding mechanism between hemostatic drugs as ligands and the human plasminogen kringle-1 (1HPK) as a target.

Conclusion: It has been suggested that SAE with tranexamic acid could be a drug-release system of microchannel transport used in MIS. This system could tackle the dilemma of fluidity and adhesion in MIS. The photo-stable tranexamic acid was the most suitable drug according to its satisfactory binding energy, good photo-stability, and sustained release.
\end{abstract}

Keywords: hemostasis, photopolymerization, minimally invasive surgery, molecular simulation

\section{Introduction}

Mild postpartum hemorrhage and long-term continuous abnormal uterine bleeding are often induced by exogenous injury and disease, such as coagulopathies or uterine atony. ${ }^{1}$ Early treatment can minimize the potentially serious outcomes of intracavitary hemorrhage.

Minimally invasive surgery (MIS) is employed widely in gynecologic therapy. ${ }^{2,3}$ Using MIS to control an intracavitary hemorrhage permits visual inspection using a hysteroscope while simultaneously minimizing the damage caused by the hemorrhage to the human body.

The blood-clotting mechanism starts from contraction of blood capillaries. Then, platelets adhere to damaged vascular walls to form a white thrombus to block bleeding from within the blood vessel. In addition, fibrin networks, through polymerized activating factors, "net" red blood cells to form a blood clot to stop bleeding from outside the blood vessel after fibrinogen hydrolysis catalyzed by thrombin., ${ }^{4,5}$

Based on the mechanism described previously, several macromolecular materials have been developed as drug carriers to control the sustained release of hemostatic agents. These materials include natural polymers (eg, alginate ester, oxycellulose, gelatin sponge, chitosan) and synthetic polymers (polylactic acid, polyvinyl alcohol [PVA], polycyanoacrylate). These polymers are used in conventional surgery submit your manuscript Dovepress If 
because of their good absorbability, biodegradability, and bio-safety (eg, chitosan is often used as a hemostatic material). However, these materials usually form a layer of film in vivo, thus they have poor liquidity and, as such, are not suitable for MIS because the surgeon must make several small incisions $(<25 \mathrm{~mm})$ and insert a trocar for the surgical procedure. Hence, low-viscosity carriers are necessary for microchannel transport.

Synthetic polymer-based carriers have the advantage of low cost, but the solid ones among them do not meet the requirement for viscosity. For example, solid polylactic acid is not suitable for passing through a micro-catheter; cyanoacrylate can cure in air, but then blocks trocars/micro-catheters. ${ }^{6}$ In particular, PVA fails to adhere to tissues even though it can exist in liquid form.

Crosslinked polymers prepared by photopolymerization in situ have been proposed to tackle the problems of fluidity and adhesion if used as drug-release carriers. It is hoped that integration of the advantages of MIS and adhesion resulting from photopolymerization may lead to better hemostasis efficiency.

6-aminocaproic acid (EACA), p-(aminomethyl) benzoic acid (PAMBA), ethylenediaminediacetic acid (EDDA), and tranexamic acid (AMCHA) are commonly used antifibrinolytic drugs..$^{7-10}$ EDDA was chosen to carry out the study although it is rarely used in clinical practice now. Appropriate crosslinked polymers loaded onto these four hemostatic drugs formed by in situ photopolymerization can be expected to release the drug in a controlled manner during MIS. However, photopolymerization is often initiated by polymerizable monomers in the presence of photoinitiators exposed to ultraviolet (UV) irradiation. Hence, the photo-stability of the drugs used for hemostasis is the key to success in MIS.

Sucrose allyl ether (SAE) is a good monomer for photopolymerization owing to its excellent biocompatibility (Figure S1). ${ }^{11}$ The sucrose group can be considered to be a safe biomaterial. If SAE is polymerized into a crosslinking membrane, the residue allyl ether can transfer into PVA if the ether group is hydrolyzed. This is analogous to the structure of PVA, which is safe if used as a pharmaceutic adjuvant. Based on its reactivity, bio-safety, and fluidity, SAE is selected as a starting hemostat used in MIS. Also, 2-hydroxy-2-methyl-1-phenyl-1-propanone (HMPP) is a very popular photoinitiator owing to its good thermal stability and miscibility with various resins. ${ }^{12}$ SAE and HMPP are liquids that are easy to mix; they can adhere to bleeding sites after photopolymerization owing to their own "stickiness". Thus, the cured film can be used as an "ideal" carrier to release the drug to exert its effect at the bleeding site.
The main mechanism for the four drugs mentioned previously is their ability to inhibit activation of fibrinogen factors, ${ }^{13}$ which allows plasminogen to not be activated and thereby inhibits fibrin dissolution. Plasminogen as the target contains five homologous domains of molecular weight of 10,000 each, known as "kringles". ${ }^{14}$ Among human plasminogen kringles (HPKs), 1HPK plays an important part as a result of the fibrin binding site in its structure. ${ }^{15-17}$ Revealing the binding mechanism between $1 \mathrm{HPK}$ and a drug embedded in SAE-based polymer carriers would be useful to establish a paradigmatic clinical pathway in the microchannel transport of hemostatic drugs.

Herein, we proposed a method to integrate the advantage of MIS and good adhesion capacity of a sugar-based polymer system via photopolymerization to obtain better hemostasis efficiency. A novel approach for evaluating the photo-stability of hemostatic drugs was established by combination of spectroscopy with quantum chemistry. Molecular docking and molecular dynamics (MD) have numerous other applications in the study of targets and ligands, whereas the independent gradient model (IGM) is considered to be novel. They were employed to simulate the binding process of a hemostatic drug to $1 \mathrm{HPK}$ to discover the binding mechanism and corresponding differences between the four drugs mentioned previously. ${ }^{18-20}$ This work will be helpful to gain a deep insight into sustained drug release for MIS for the purpose of hemostasis.

\section{Materials and methods Chemicals and reagents}

HMPP was purchased from BASF Specialty Chemicals Co., Ltd., (Ludwigshafen, Germany) and purified by distillation. HMPP purity was assessed by elemental analyses. EACA, PAMBA, EDDA, and AMCHA were analytical reagents and obtained from MedTrade Products Ltd. (Crewe, UK), without further treatment. SAE was synthesized according to the method of Yang et al. ${ }^{21}$ The spectral characterization and kinetics of SAE have been shown in our early work. ${ }^{22}$ Methanol and acetonitrile are chromatographic reagents and were obtained from Aladdin Industrial Corporation, Shanghai, China. Chitosan hemostatic powder was obtained from MedTrade Products Ltd.

\section{Spectroscopy and viscosity experiments}

A high-intensity mercury-xenon lamp (LC8 L9588; Hamamatsu Photonics, Hamamatsu, Hapan) as a point light source was used to illuminate, cumulatively, the sample when UV/ vis absorption was measured on a spectrometer (8453; Agilent Technologies, Santa Clara, CA, USA) using double-distilled 
water as solvent. The viscosity data of the SAE monomer were obtained using a rotational rheometer (Kinexus Pro; Malvern Instruments, Malvern, UK) at room temperature and atmospheric pressure. The adhesive force of poly(sucrose allyl ether) (PSAE) was obtained by TA.XT Plus (Stable Micro Systems, Ltd., Goldalming, UK). A Fourier transform infrared system (7600; Thermo Fisher Scientific, Waltham, MA, USA) was used to carry out real-time infrared (RT-IR) experiments with light intensity of $38 \mathrm{~mW} / \mathrm{cm}^{-2}$ (LC8 L9588; Hamamatsu). The reaction was monitored by following the change in the $\mathrm{C}=\mathrm{C}$ stretching vibration peak at $1,645 \mathrm{~cm}^{-1}$ corresponding to an allyl group. The peak was different from the stretching band of the acrylate $(\mathrm{C}=\mathrm{C})$ and carbonyl $(\mathrm{C}=\mathrm{O})$ double bonds positioned at $1,625 \mathrm{~cm}^{-1}$ and $1,658 \mathrm{~cm}^{-1}$, respectively. ${ }^{23}$

\section{Drug-release experiments in vitro}

SAE, HMPP, and drugs were mixed well and exposed to a spot light source (xenon lamp) for photopolymerization within 1.0 minute. The amount of each formulation containing SAE, HMPP, and drugs had a weight ratio of 30.0/2.0/5.0 (30.0/2.0/1.0 for PAMBA). The formed membrane was extracted by double-distilled water at $30^{\circ} \mathrm{C}$ without stirring. A small amount of solution was extracted at regular intervals, and then measured by high-performance liquid chromatography (HPLC) to detect the drug concentration using an LC-20A system with an Inertsil ODS-3 column (both from Shimadzu, Kyoto, Japan) at a flow rate of $1.0 \mathrm{~mL} \cdot \mathrm{min}^{-1}$ and column temperature of $30^{\circ} \mathrm{C}$. Measurements were carried out with an injection volume of $20.0 \mu \mathrm{L}$ and a detection wavelength of $208 \mathrm{~nm}$ for EACA and AMCHA, $220 \mathrm{~nm}$ for PAMBA, and $254 \mathrm{~nm}$ for EDDA. The mobile phase was a mixture of acetonitrile with $1.0 \%$ phosphate buffer (10/90) containing $0.055 \%$ of sodium hexanesulfonate (prepared by addition of $0.055 \%$ sodium hexanesulfonate and phosphoric acid at $\mathrm{pH}$ 2.2) for EACA and AMCHA; methanol/phosphate buffer (pH 2.5) (40/60) for PAMBA; acetonitrile with $0.06 \%$ tetrabutylammonium hydroxide ( $\mathrm{pH} 6.5$ with phosphoric acid) solution (20/80) for EDDA.

\section{Hemostasis experiments in vivo}

A hemostasis model was established in vivo. Ten healthy adult Sprague-Dawley rats (180-200 g; Guangdong Medical Laboratory Animal Center) were divided randomly and equally into two groups: muscle and liver. Then, 3\% pentobarbital sodium (45 mg/kg body weight, intraperitoneally) was injected into all rats before experimentation.

In the muscle group, rats were fixed prone after the induction of anesthesia. The skin was incised, and the left and right gluteus maximus cut; a cross-shaped wound of
$2.0 \times 1.0 \mathrm{~cm}$ was made using a scalpel. After that, a mixture of SAE, HMPP, and hemostatic drugs was applied to the wound surface, and then it was exposed to a spot light source for 1.0 minute.

In the liver group, rats were fixed supine after anesthesia induction. A 2.0-cm longitudinal incision was made under the ribs and abdominal muscles cut along the abdominal line. Then, the abdomen was squeezed and the liver delivered from the incision. A cross-shaped wound of $1.0 \times 1.0 \mathrm{~cm}$ was made at the center of the liver surface. A mixture of SAE, HMPP, and hemostatic drugs was applied to the wound surface, and then it was exposed to a spot light source for 1.0 minute.

The Animal Ethics Committee of Guangzhou Medical University approved the experiment, and the guidelines on the care and use of laboratory animals of the committee were strictly followed.

\section{Cytotoxicity experiments}

HMPP was purified by distillation to obtain a colorless liquid and was characterized by elemental analyses. SAE was mixed with $6.0 \%$ HMPP and polymerization initiated by a point light source. The formed membrane was immersed in a culture medium containing $0.1 \mathrm{~g} / \mathrm{mL}$ of serum at $37^{\circ} \mathrm{C}$ for 24 hours and 72 hours after sterilization according to ISO 10993-5. Then, the supernatant was taken as an extract. L929 cells and BRL $3 \mathrm{~A}$ cells were selected to evaluate cytotoxicity. Then, $100 \mu \mathrm{L}$ of the medium and 10\% FBS (containing $\sim 10^{4}$ cells) were seeded into the wells of a 96-well plate and incubated under standard culture conditions to adhere cells to the plate. Subsequently, $10 \mu \mathrm{L}$ of the extract was added to each well after incubation for 24 hours. The negative control group was treated with $10 \mu \mathrm{L}$ of an extract of high-density polyethylene $(0.1 \mathrm{~g} / \mathrm{mL})$ as a reference. The positive control group was treated with $10 \mu \mathrm{L}$ of phenol solution $(0.1 \mathrm{~g} / \mathrm{mL})$. The blank control group was treated with serum medium. Then, $10 \mu \mathrm{L}$ of Cell Counting Kit-8 (CCK-8) was added to the experimental hole after 4, 24, 48, and 72 hours, respectively. Absorbance was recorded (Epoch; BioTek, Winooski, VT, USA) after incubation for 4 hours.

\section{Calculation of the excited state}

The optimization and frequency of the ground state and excited state of hemostatic drugs were calculated according to the time-dependent density functional theory (TD-DFT) using the M062x functional and 6-311++g(d,p) basis set in Gaussian 2016. ${ }^{24}$

\section{Molecular docking}

The three-dimensional (3D) geometry of the four hemostatic drugs was optimized in Gaussian 2016 before molecular 
docking. The crystallographic structure of the 1HPK domain (Protein Data Bank [PDB] id: 1HPK) and EACA complex were downloaded from the PDB of the Research Collaboratory for Structural Bioinformatics. ${ }^{25}$ Because it contains EACA inside, after removing EACA, the structure of the protein chain remaining was taken to be the target receptor. The docking of the four drugs with 1HPK was done using Auto Dock 4.2. ${ }^{26,27}$ Here, $1 \mathrm{HPK}$ was a rigid receptor whereas the four drugs were treated as flexible ligands by unfreezing all the torsional bonds at their input conformations without torsional constraints. Gasteiger charges were computed for $1 \mathrm{HPK}$ and drugs. The grid box was set as $40 \times 40 \times 40$ grid points with a resolution of $0.375 \AA$ and each obtained ten independent docking calculations, respectively. The conformation with lowest binding energy was selected as the most favorable conformation for each docking complex.

\section{MD simulations}

The initial conformations for MD simulations were from the results of docking. MD simulations of $2.0 \mathrm{~ns}$ were undertaken with Gromacs 5.0.5 and the optimized potentials for liquid simulations all-atom model (OPLS-AA) force field to study the dynamic stability of the four docking complexes. ${ }^{28-30}$ Here, 1HPK and drugs were separated from the docking complex to prepare a topology file, respectively. The docking complex was solvated in a cubic box with a blank of $\geq 1.0 \mathrm{~nm}$ from the box edge (SPC/E water model), and then neutralized by adding sodium ions or chloride ions. The SPC/E water model specifies a 3-site rigid water molecule with LennardJones parameters and partial charge assignments change for $\mathrm{O}(-0.8476)$ and $\mathrm{H}(0.4238)$ assigned to each of the three atoms. The position of the protein was restrained with a force constant of 1,000 . The system was then minimized with 50,000 steps at steepest descent minimization and positionrestrained dynamics. Then, 50,000 steps and 1,000,000 steps were taken using the Nose-Hoover method at $300 \mathrm{~K}$ and using the Parrinello-Rahman method at $1.0 \mathrm{~atm}$, respectively. Finally, MD simulations of 2.0 ns were undertaken until the system reached equilibrium.

\section{Calculation of binding free energy}

Calculations of binding free energy were undertaken by the tool g_mmpbsa and gmxpbsatool, which implemented the molecular mechanics-Poisson Boltzmann surface area (MM-PBSA) approach using subroutines written in-house or sourced from Gromacs and Apbs packages. ${ }^{31-33}$ For each complex, 250 snapshots isolated from the last $1 \mathrm{~ns}$ simulation were used to calculate the binding free energy. Schematic diagrams of protein-ligand interactions were generated by Ligplot. ${ }^{34}$ The source of MM-PBSA was from Gromacs 4.6.7. The meaning of subitems is shown in Equations 1-3:

$$
\begin{gathered}
\Delta \mathrm{G}_{\text {bind }}=\Delta \mathrm{E}_{\mathrm{MM}}+\Delta \mathrm{G}_{\text {solv }}-\mathrm{T} \Delta \mathrm{S} \\
\Delta \mathrm{G}_{\text {solv }}=\Delta \mathrm{G}_{\text {polar }}+\Delta \mathrm{G}_{\text {non-polar }} \\
\Delta \mathrm{E}_{\mathrm{MM}}=\Delta \mathrm{E}_{\mathrm{vdw}}+\Delta \mathrm{E}_{\text {ele }}
\end{gathered}
$$

where $\Delta \mathrm{G}_{\text {bind }}$ is the total molecular mechanics energy in the gas phase, $\Delta \mathrm{G}_{\text {solv }}$ is a correction term (solvation free energy) of each system surrounded by solvent, and $\mathrm{T} \Delta \mathrm{S}$ is the entropy. $\Delta \mathrm{E}_{\mathrm{MM}}$ includes electrostatic $\Delta \mathrm{E}_{\mathrm{ele}}$, and Van der Waals $\Delta \mathrm{E}_{\mathrm{vdw}}$ energies, whereas $\Delta \mathrm{G}_{\text {solv }}$ is the sum of electrostatic solvation energy $\Delta \mathrm{G}_{\text {polar-bind }}$, and the non-electrostatic solvation component $\Delta \mathrm{G}_{\text {non-polar }}$. The polar contribution was calculated using the Poisson-Boltzmann model, whereas non-polar energy was estimated by solvent-accessible surface area. In this study, the $\mathrm{T} \Delta \mathrm{S}$ term was excluded.

\section{Weak interaction between ligands and proteins}

Description of a weak interaction is dependent upon density gradient function (reduced density gradient [RDG]), and the arithmetic expression is shown in Equation $4:^{35}$

$$
\mathrm{RDG}=\frac{1}{2 \sqrt[3]{3 \pi^{2}}} \cdot \frac{|\nabla \rho(r)|}{\sqrt[3]{\rho(r)^{4}}}
$$

Usually, $|\nabla \rho(r)| / \sqrt[3]{\rho(r)^{4}}$ is a focus, where $\nabla$ is the gradient operator, $\mathrm{r}$ is radius, and $|\nabla \rho(r)|$ is the modulus of the electron density gradient. Data were obtained by IGMPlot according to the IGM. ${ }^{36}$ The weak interaction of intermolecular, $\delta_{g_{-} \text {inter }}$, can be obtained:

$$
\left(\frac{\partial \rho}{\partial x}\right)^{\mathrm{IGM}, \text { inter }}=\left|\sum_{i=1}^{N_{A}} \frac{\partial \rho_{i}}{\partial x}\right|+\left|\sum_{i=1}^{N_{B}} \frac{\partial \rho_{i}}{\partial x}\right|
$$

\section{Results}

\section{UV photolysis and calculations of the excited state}

Experiments on UV photolysis were used to evaluate the photo-stability of the four drugs, and their absorption spectra under increasing irradiation are shown in Figure 1. The maximum absorption peak $\left(\lambda_{\max }\right)$ was $196 \mathrm{~nm}$ for EACA, $195 \mathrm{~nm}$ 

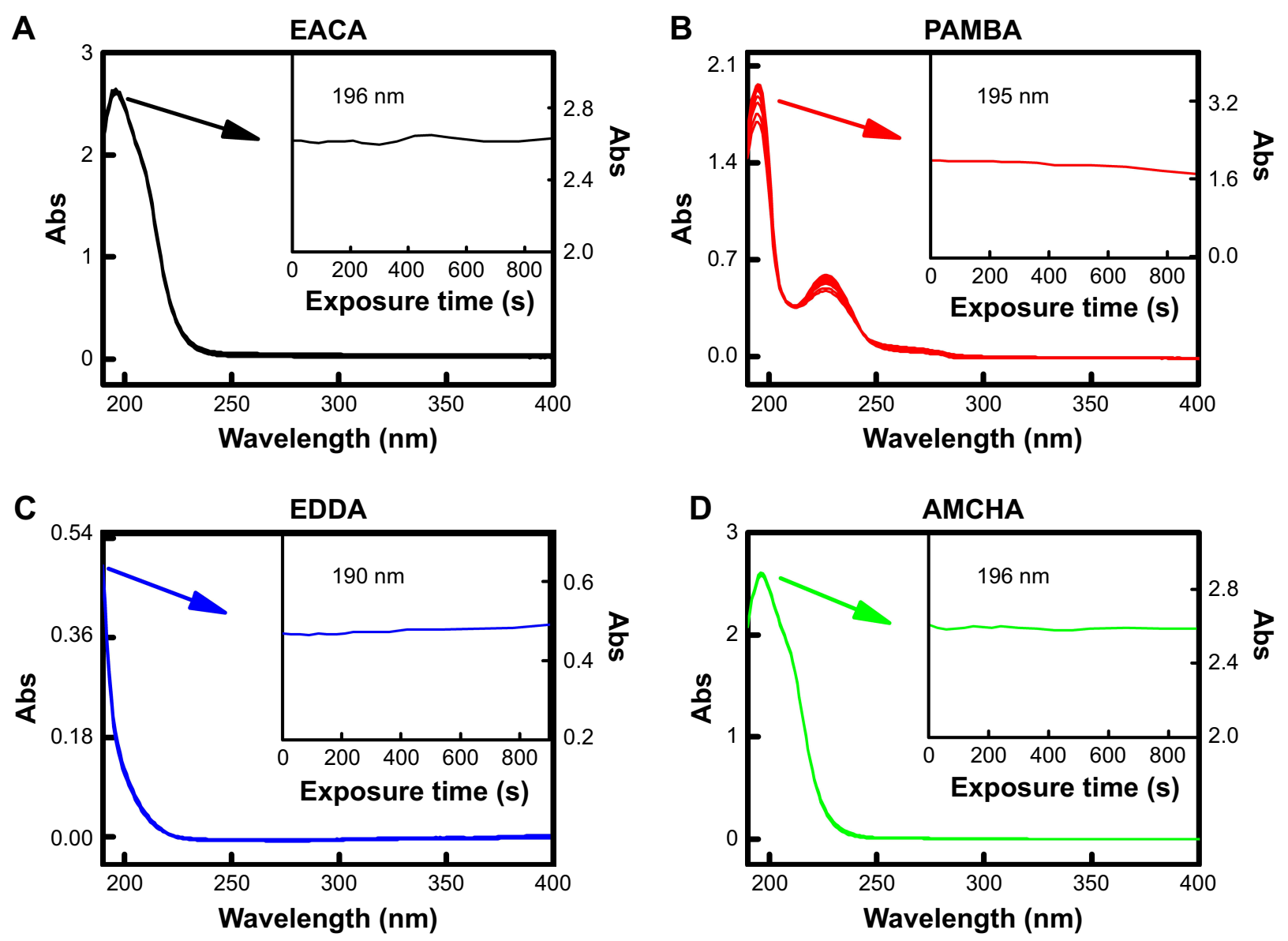

Figure I UV photolysis spectra of four hemostatic drugs with changing exposure time, respectively (A) EACA, (B) PAMBA, (C) EDDA, and (D) AMCHA, inset: change of $\lambda_{\max }$ as a function of the steady-state exposure time.

Note: Drug concentration: $0.001 \mathrm{~mol} / \mathrm{L}$ (EACA and AMCHA), $6.0 \times 10^{-5} \mathrm{~mol} / \mathrm{L}$ (PAMBA) and 5.0 $\times 10^{-4} \mathrm{~mol} / \mathrm{L}$ (EDDA).

Abbreviations: Abs, absorbance; AMCHA, tranexamic acid; EACA, 6-aminocaproic acid; EDDA, ethylenediaminediacetic acid; PAMBA, p-(aminomethyl) benzoic acid.

for PAMBA, and $196 \mathrm{~nm}$ for AMCHA (Figure 1A, B and D), but EDDA did not show $\lambda_{\text {max }}>190 \mathrm{~nm}$ (Figure 1C). Insets of Figure 1A, C, and D also show that EACA, EDDA, and AMCHA were stable under UV light because absorbance at the $\lambda_{\max }$ for them (the chosen wavelength was $190 \mathrm{~nm}$ for EDDA) changed little within a UV-irradiation time of 900 seconds. PAMBA could be viewed as a quasi-photostable drug because absorbance at its $\lambda_{\max }$ was constant within 400 seconds (Figure 1B inset), and it decreased after a longer irradiation time. According to UV-photolysis determinations, an irradiation time of 1.0 minute was chosen for polymerization of a mixture of SEA/HMPP/drug to avoid possible side reactions in subsequent experiments on drug release and creation of a hemostasis model.

TD-DFT simulation was done to assess the reason for the photo-stability of the test drugs. When a molecule is excited to the singlet state $(\mathrm{S})$ from the ground state after absorbing light energy, it transfers to the triplet state $(\mathrm{T})$ through an intersystem-crossing process. The excitation energies of the four drugs are shown in Table 1. The vertical excitation energy $\left(\mathrm{E}_{\mathrm{vert}}\right)$ is the energy which only electrons are excited to a higher energy orbital (Franck-Condon point) from a ground state (lowest point) without a change in conformation or configuration (Figure S2). The adiabatic excitation energy $\left(\mathrm{E}_{\text {adiab }}\right)$ is the energy difference between the lowest point of the excited state and lowest point of the ground state. $\mathrm{E}_{\text {adiab }}$ involves a change in conformation or configuration in the potential energy surface (PES) of the excited state because it needs to reach the most stable structure. The relaxation energy $\left(\mathrm{E}_{\text {relax }}\right)$ is obtained from the energy change between the Franck-Condon point and the lowest point in the PES of the excited state $\left(\mathrm{E}_{\text {relax }}{ }^{\mathrm{ES}}\right)$. It can also be obtained from the energy change between the ground zero of the lowest point in the excited state and lowest point in the PES of the ground state $\left(\mathrm{E}_{\text {relax }} \mathrm{GS}\right)$. The reorganization energy $\left(\mathrm{E}_{\text {reorg }}\right)$ in Table 1 is a good indicator of photo-stability and is the sum of $\mathrm{E}_{\text {relax }}{ }^{\mathrm{ES}}$ and $\mathrm{E}_{\text {relax }}{ }^{\mathrm{GS}}$. The $\mathrm{E}_{\text {reorg }}{ }^{\text {total }}$ is the sum of $\mathrm{E}_{\text {reorg }}{ }^{\mathrm{S} 1}$ and $\mathrm{E}_{\text {reorg }}{ }^{\mathrm{T} 1}$. A drug can be considered to be photo-stable if its 
Table I The energies of photo-stable drugs calculated from the TD-DFT

\begin{tabular}{|c|c|c|c|c|c|c|c|}
\hline \multirow[t]{2}{*}{ Drug } & \multirow[t]{2}{*}{ Excited state } & $\mathbf{E}_{\text {vert }}$ & $E_{\text {adiab }}$ & $\mathbf{E}_{\text {relax }}$ ES & $E_{\text {relax }}{ }^{G S}$ & $E_{\text {reorg }}$ & $\mathbf{E}_{\text {reorg }}^{\text {total }}$ \\
\hline & & $\mathrm{kcal} / \mathrm{mol}$ & $\mathrm{kcal} / \mathrm{mol}$ & $\mathrm{kcal} / \mathrm{mol}$ & kcal/mol & kcal/mol & $\mathrm{kcal} / \mathrm{mol}$ \\
\hline \multirow[t]{2}{*}{ EACA } & SI & $|32.4|$ & 73.53 & 58.88 & 37.81 & 96.68 & \multirow{2}{*}{203.48} \\
\hline & $\mathrm{TI}$ & 122.29 & 57.61 & 64.68 & 42.12 & 106.80 & \\
\hline \multirow[t]{2}{*}{ PAMBA } & SI & 116.15 & 86.37 & 29.78 & 13.85 & 43.63 & \multirow{2}{*}{97.27} \\
\hline & $\mathrm{TI}$ & 91.91 & 59.06 & 32.86 & 20.78 & 53.64 & \\
\hline \multirow[t]{2}{*}{ EDDA } & SI & 134.08 & 74.42 & 59.66 & 44.11 & 103.77 & \multirow{2}{*}{224.77} \\
\hline & TI & 124.11 & 54.81 & 69.30 & 51.71 & 121.00 & \\
\hline \multirow[t]{2}{*}{$\mathrm{AMCHA}$} & SI & 127.96 & 74.20 & 53.76 & 31.00 & 84.76 & \multirow{2}{*}{179.12} \\
\hline & $\mathrm{TI}$ & 117.49 & 58.31 & 59.18 & 35.18 & 94.36 & \\
\hline
\end{tabular}

Abbreviations: $A M C H A$, tranexamic acid; $E A C A, 6$-aminocaproic acid; $\mathrm{E}_{\text {adia, }}$, adiabatic excitation energy; $E_{D D A}$, ethylenediaminediacetic acid; $\mathrm{E}_{\text {relax }}$, relaxation energy; $\mathrm{E}_{\text {reorg, }}$ reorganization energy; $\mathrm{E}_{\mathrm{vert}}$, vertical excitation energy; ES, excited state; GS, ground state; PAMBA, P-(aminomethyl) benzoic acid; SI, first singlet state; TI, first triplet state; TD-DFT, time-dependent density functional theory.

$\mathrm{E}_{\text {reorg }}^{\text {total }}$ is $>120 \mathrm{kcal} \cdot \mathrm{mol}^{-1}$, but $\mathrm{E}_{\text {reorg }}^{\text {total }}$ for a photosensitive drug is $<60 \mathrm{kcal} \cdot \mathrm{mol}^{-1}$. $^{37}$ Thus, the hemostatic drugs EACA, EDDA, and AMCHA were photo-stable because their total reorganization energies were $>120 \mathrm{kcal} \cdot \mathrm{mol}^{-1}$. However, the $\mathrm{E}_{\text {reorg }}{ }^{\text {total }}$ of PAMBA was $60-120 \mathrm{kcal} \cdot \mathrm{mol}^{-1}$ $\left(97.27 \mathrm{kcal} \cdot \mathrm{mol}^{-1}\right)$, suggesting that PAMBA was a quasiphoto-stable drug. Hence, the theoretical calculation and UVphotolysis experiments could explain the photo-stability of these hemostatic drugs reasonably well. These results showed that at least three hemostatic drugs could be used in MIS.

\section{Viscosity measurements}

To clarify the mobility of SAE liquid in the trocars used in MIS, its viscosity under different shear rates and temperature was measured (Figure 2). For comparison, the viscosity of 2-hydroxyethyl methacrylate (HEMA) liquid, a common monomer used widely to form polymers in biomaterials (eg, contact lenses), is also shown in Figure 2. The viscosity of SAE decreased with increasing shear rate and showed

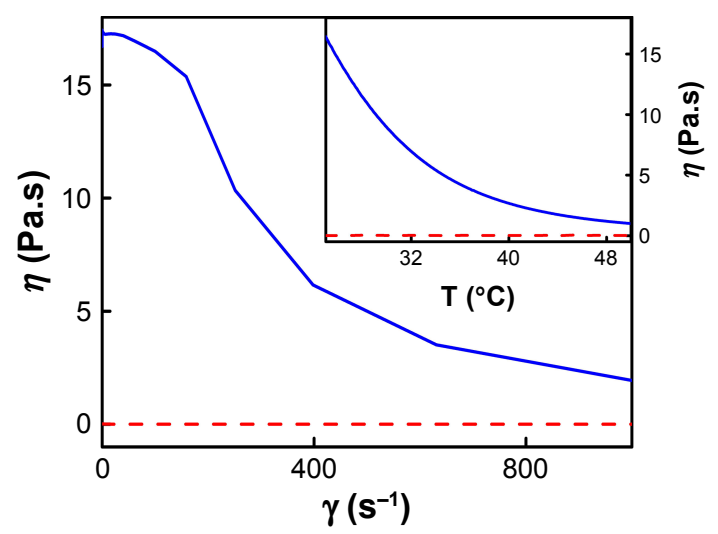

Figure 2 The viscosity of SAE (-) and HEMA (-- ).

Notes: Viscosity vs shearing rate $\left(\right.$ at $\left.37^{\circ} \mathrm{C}\right)$; inset: viscosity vs temperature at shearing rate (I Pa.s).

Abbreviations: HEMA, 2-hydroxyethyl methacrylate; SAE, sucrose allyl ether. shear-thinning behavior. The viscosity of SAE also decreased with increasing temperature from $25^{\circ} \mathrm{C}$ to $50^{\circ} \mathrm{C}$. However, the viscosity of HEMA was almost unchanged with increasing shear rate and temperature in the same ranges, which suggested that the viscosity of HEMA was so low that it could not be detected. Sucrose is a solid, but allyl substitution of the hydroxyl groups of sucrose for preparation of SAE weakens hydrogen bonds, leading to the lower viscosity of SAE. This rheological feature of SAE facilitates drug transport in microchannels because the formulation can pass through a sheath tube by adding mild pressure in MIS. The mobility of sucrose oleate ester was also investigated for comparison with SAE (data not shown) because both are liquids and have the same sucrose core. ${ }^{22}$ Sucrose oleate ester displayed similar rheological behavior to that of HEMA, but sucrose oleate ester was not polymerizable in the presence of photoinitiators.

\section{RT-IR experiments}

RT-IR was used to determine the photopolymerization kinetics of the SAE monomer so that clinical viability could be evaluated. Oxygen inhibition could be ignored because $\mathrm{CO}_{2}$ is often used in MIS (eg, enteroscopy). HMPP was used to initiate the photopolymerization of SAE to form PSAE film while the HMPP content varied from $2 \%$ to $8 \%$ (Figure 3). The polymerization rate (determined by the slope of the plot) increased with increasing HMPP concentration within an initial irradiation time of 120 seconds and slowed down after exceeding 200 seconds. The sharp rate at the beginning of polymerization indicated typical auto-acceleration of the bulk polymerization reaction. Conversion of $\mathrm{C}=\mathrm{C}$ double bonds increased with increasing irradiation time independent of the HMPP concentration. Conversely, a higher HMPP content did not enhance the final conversion. This was evidenced by conversion at $8 \mathrm{wt} \%$ of HMPP, which was lower than the conversion at 


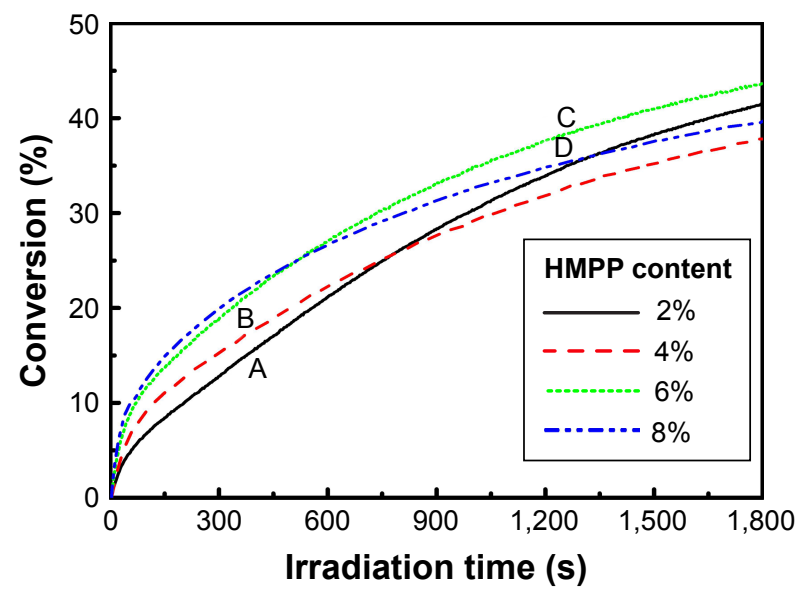

Figure 3 Photopolymerization kinetics at different HMPP concentrations with the light intensity of $38 \mathrm{~mW} / \mathrm{cm}^{-2}$.

Notes: (A) 2 wt\%, (B) 4 wt\%, (C) 6 wt\%, and (D) 8 wt\%. HMPP concentration was expressed as the mass percentage of the SAE.

Abbreviations: HMPP, 2-hydroxy-2-methyl-I-phenyl-I-propanone; SAE, sucrose allyl ether.

$2.0 \mathrm{wt} \%$ of HMPP after 600 seconds. This effect may have been due to extra free radicals that caused early termination of the chain reaction. Based on the highest polymerization rate and consideration of bio-safety, $6 \mathrm{wt} \%$ of HMPP was selected to achieve the optimal polymerization conditions used in subsequent experiments.

\section{Tissue adherence}

The adhesive force of PSAE is more than its crosslinking force, which makes measurement of the tissue adherence of PSAE difficult. PSAE is a fragile material, so we used cell adhesion percentages to describe the adhesive force instead of the pull test. We found the portion of adhesion in the sum of adhered and suspended cells to be $70 \%-80 \%$. A detailed test with a meat product (bacon) was done in a subsequent experiment. We also detected the adhesive force of a membrane adhering to the bacon, and a quartz glass was placed on the bacon. After irradiation by a UV spot light source with light intensity of $48 \mathrm{~mW} / \mathrm{cm}^{-2}$ for 1.0 minute, the maximum adhesive force of PSAE on the surface of the quartz glass measured was $\sim 40 \mathrm{~g}$. Unfortunately we could not obtain a suitable result for the adhesive force from the bacon at present.

\section{Sustained release experiments in vitro}

To verify the feasibility of using a crosslinked PSAE matrix as a drug carrier, sustained-release experiments in vitro were done by HPLC. Figure 4 presents the amount of drug released as a function of extraction time. All drugs were released within 5 days, and EACA and PAMBA were released faster than AMCHA and EDDA at the beginning of 6 days.

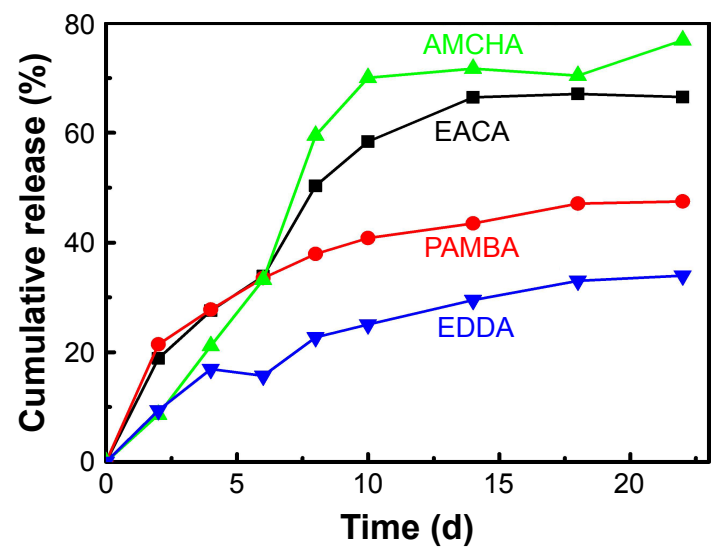

Figure 4 The cumulative release percentage of the drug within 20 days. Notes: $\operatorname{EACA}(\boldsymbol{\nabla}), \operatorname{PAMBA}(\bullet), \operatorname{EDDA}(\boldsymbol{\nabla})$ and AMCHA $(\boldsymbol{\Delta})$

Abbreviations: AMCHA, tranexamic acid; EACA, 6-aminocaproic acid; EDDA, ethylenediaminediacetic acid; PAMBA, p-(aminomethyl) benzoic acid.

In addition, the final amount of EACA and AMCHA release was $>70 \%$ after 20 days, whereas it was $45 \%$ and $30 \%$ for PAMBA and EDDA, respectively. EACA and AMCHA could be dissolved in water readily but PAMBA and EDDA could not. Thus, the final amount of drug released was determined by its solubility in water. Also, drug release was a long process until a final equilibrium was reached. Hence, the crosslinked PSAE film containing embedded hemostatic drugs could be used as a drug carrier and allowed the hemostatic drugs to exert long-term effects.

\section{Cytotoxicity}

Cytotoxicity of SAE and HMPP was assessed by the CCK-8 assay and was expressed as the half-maximal inhibitory concentration $\left(\mathrm{IC}_{50}\right)$ (Figure 5). The measured $\mathrm{IC}_{50}$ (in $\mu \mathrm{g} \cdot \mathrm{mL}^{-1}$ )

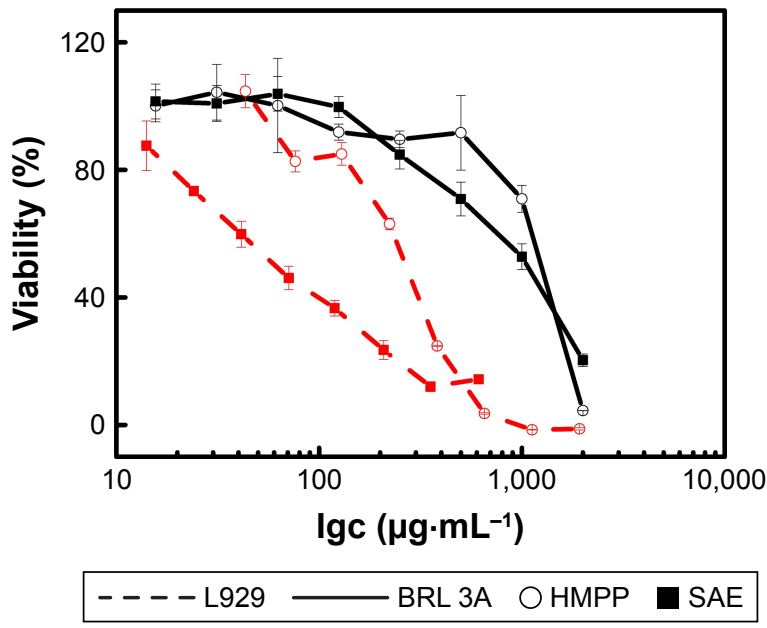

Figure 5 The cytotoxicity of SAE (ロ) and HMPP (O) for L929 cells (-) and BRL $3 \mathrm{~A}$ rat hepatocytes $(---)$ by using the CCK-8 assay.

Abbreviations: CCK-8, Cell Counting Kit-8; HMPP, 2-hydroxy-2-methyl- I-phenylI-propanone; SAE, sucrose allyl ether. 
of SAE and HMPP was 929 and 1,054 for L929 cells, and 693 and 1,349 for BRL 3A cells, respectively. The photoinitiator Sarcat ${ }^{\mathrm{TM}} \mathrm{CD} 1,012$ has an $\mathrm{IC}_{50}$ of $14 \mu \mathrm{g} \cdot \mathrm{mL}^{-1}$ to human monocytes. ${ }^{38,39}$ Thus, SAE and HHMP showed satisfactory bio-safety. As a commonly used solid photoinitiator, 1-[4-(2-Hydroxy)-phenyl]-2-hydroxy-2-methyl-1-propane1-one (Irgacure 2959) had better bio-safety. Unfortunately, it cannot be used in MIS because it needs a long time to dissolve in the SAE monomer. PSAE was safe to use because it can change into a polyallyl alcohol structure after hydrolysis, and polyallyl alcohol is a PVA analog, which has been used widely in MIS for a long time.

\section{Hemostasis experiments in vivo}

Previously, we showed that a solid film formed by photopolymerization with SAE and HMPP can be used for hemostasis for a short time. The blood-clotting mechanism, including the corresponding statistical data, were also discussed. ${ }^{22}$ Here, we used the solid film to "carry" hemostatic drugs to the bleeding site for long-term hemostasis. Like before, a hemostasis model in vivo was established to apply in MIS. A mixture of SAE, HMPP, and hemostatic drug was applied to cover a bleeding wound and then irradiated by UV light to initiate polymerization. In contrast with as-prepared drug-loaded carriers, chitosan hemostatic powder was chosen. Chitosan is a popular material used directly for hemostasis without irradiation owing to its acceptable biodegradability and satisfactory biocompatibility. The hemostasis results for the test drugs are shown in Figure $6 .^{40,41}$ A small amount of blood flowed when the muscle was cut. The liquid mixture of SAE, HMPP, and hemostatic drugs quickly covered the bleeding wound, and then the mixture was exposed to UV light to initiate polymerization for 1.0 minute. The liquid formulation was converted gradually to a solid film under UV light. A closed space was formed between the solid film and muscle tissues (Figure 6), which denoted visible hemostasis at this stage. In a preliminary study, we found that the bleeding time without a drug was slightly longer than that with a drug. We also found the formed film containing EACA (Figure 6A) or EDDA (Figure 6C) to be transparent, whereas the film containing PAMBA or AMCHA was opaque to some extent. The opacity of the two films was caused by the hemostatic drug because the film without a hemostatic drug was transparent. The transparent film would aid observation of the hemostasis effect of the released drugs during MIS. For chitosan, rapid accumulation of blood around a wound led to formation of a gel-like layer of protective membrane when the hemostatic powder was applied to a bleeding wound. The scenarios in the liver group were identical to those in the muscle group. By establishing a hemostasis model in vivo,
A
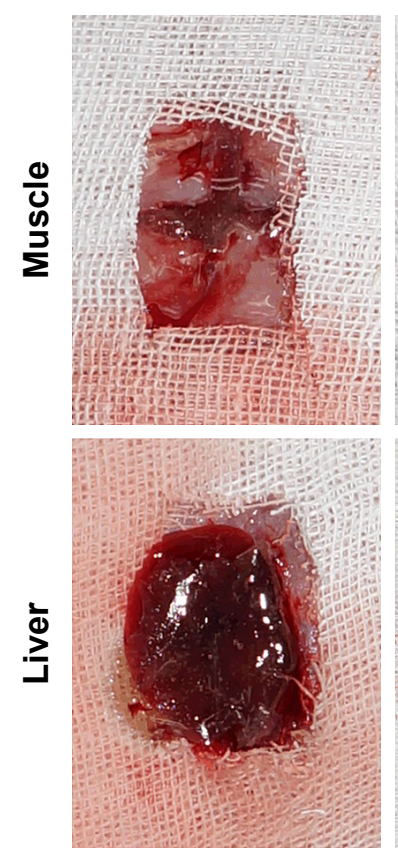

B

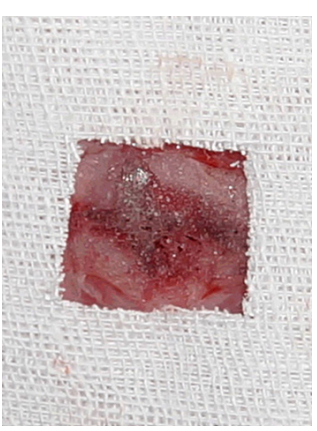

C
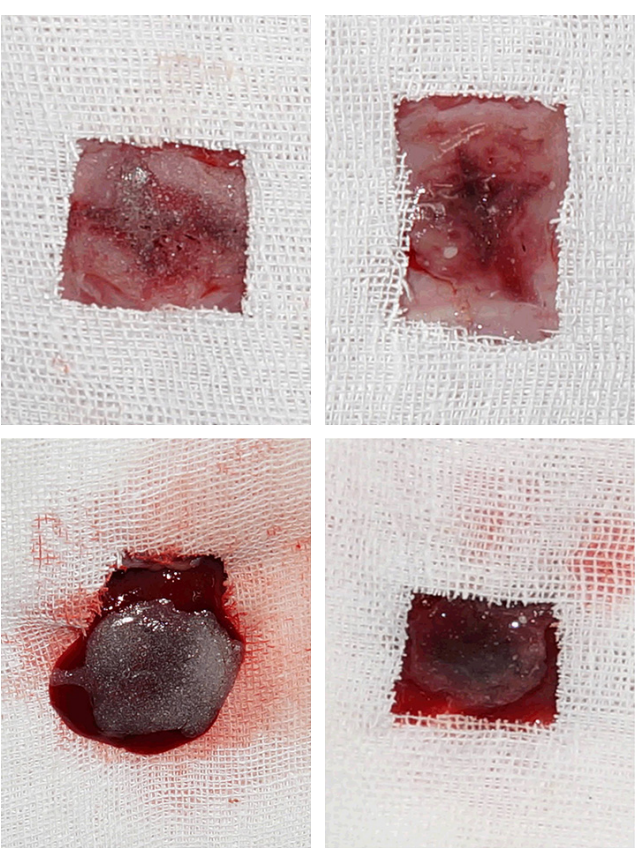

D

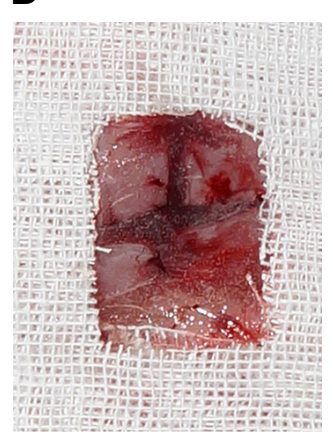

E
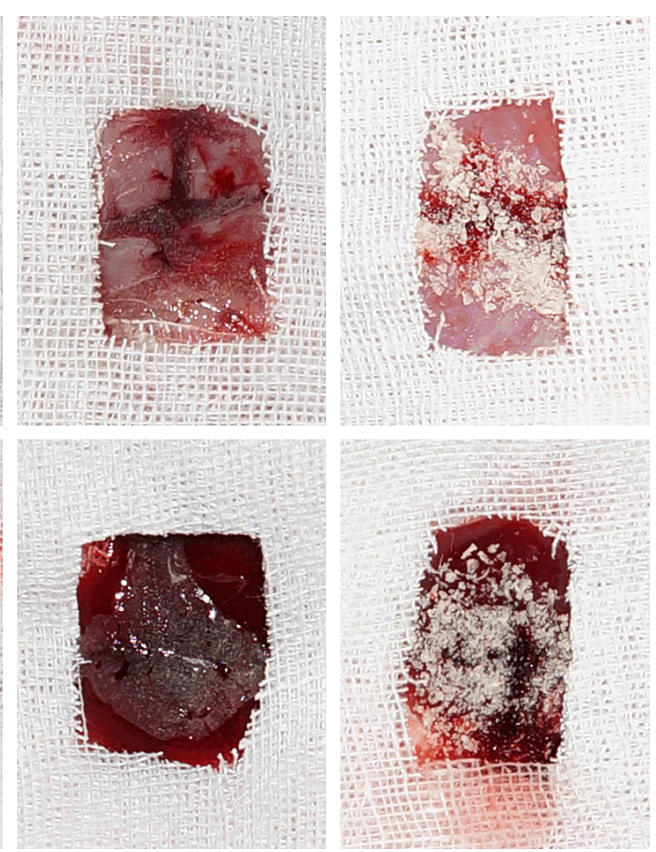

Figure 6 The hemostasis models of the drug of (A) EACA, (B) PAMBA, (C) EDDA, (D) AMCHA, and (E) chitosan powder.

Abbreviations: AMCHA, tranexamic acid; EACA, 6-aminocaproic acid; EDDA, ethylenediaminediacetic acid; PAMBA, p-(aminomethyl) benzoic acid. 
we showed that a crosslinked sugar-based polymer film could be used as a hemostatic drug carrier, which could provide useful clinical applications in the future.

\section{Molecular docking}

Molecular simulations were carried out to ascertain the most suitable hemostatic drug through the binding mechanism between the drug and 1HPK protein. The force field of autodock was effective for prediction of the binding energy. The binding energy $\left(\Delta \mathrm{E}_{\text {bind }}\right)$ of the drug/1HPK complex (which reflects affinity) was obtained from the results of molecular docking shown in Table 2. Three major contributions to $\Delta \mathrm{E}_{\text {bind }}$ were $\Delta \mathrm{E}_{\mathrm{vdw} \_ \text {hb_desolv }}$ (sum of Van der Waals energy, hydrogen bond energy, and desolvation energy), $\Delta \mathrm{E}_{\text {ele }}$ of electrostatic energy, and $\Delta \mathrm{E}_{\text {torsional }}$ of torsional energy, also listed in Table 2. The decrease in $\Delta \mathrm{E}_{\text {bind }}$ was in the order AMCHA $>$ PAMBA $>$ EACA $>$ EDDA. The docking complex of AMCHA/ $1 \mathrm{HPK}$ had a maximum $\Delta \mathrm{E}_{\text {bind }}$ of $-20.785 \mathrm{~kJ} \cdot \mathrm{mol}^{-1}$, which indicated that the affinity of AMCHA binding to $1 \mathrm{HPK}$ was the strongest. $\Delta \mathrm{E}_{\mathrm{vdw} \_ \text {hb_desolv }}$ and $\Delta \mathrm{E}_{\text {ele }}$ made favorable contributions to $\Delta \mathrm{E}_{\text {bind }}$, resulting in increasing stability of the docking models, but $\Delta \mathrm{E}_{\text {torsional }}$ made unfavorable contributions. EDDA was considered to increase the potential of specific molecular recognition because it had the highest $\Delta \mathrm{E}_{\text {torsional }}\left(11.208 \mathrm{~kJ} \cdot \mathrm{mol}^{-1}\right)$. However, EDDA had the lowest $\Delta \mathrm{E}_{\mathrm{vdw} \_ \text {hb_desolv }}$ and $\Delta \mathrm{E}_{\text {ele }}$ (negative value) among the four drugs, and was considered to have unfavorable charge-charge interactions.

\section{MD simulations}

Molecular-docking results often vary depending on molecular flexibility because inadequate conformational searching and flawed scoring can increase errors. To ascertain the correct positions in a more precise way, 2 ns of MD simulations were carried out for the four types of complexes (Figure 7). The root mean square distance (RMSD) of the four complexes of drug/1HPK is shown in Figure 7A. Fluctuation trends in the four complexes were similar to each other, and the four systems reached equilibrium after $1.5 \mathrm{~ns}$. The final RMSD values for all complexes changed considerably, suggesting that ligand and target receptors were arranged in a more compact and stable mode. The RMSD of the complex of EDDA/1HPK had the largest fluctuation among the four docking complexes, showing that this complex was the most unstable one before equilibrium had been reached, but the complex of PAMBA/1HPK was the most stable one. In addition, the results of steered molecular dynamics (SMD) are shown in Figure 7B, where artificial dissociation was considered to be the inverse process of docking. Typically, for the departure process of PAMBA, one platform was found at a $\xi$ of $\sim 1.5 \mathrm{~nm}$, and a remarkable feature was that the potential of mean force (PMF) curve started with a $\xi$ of $0 \mathrm{~nm}$, which

Table 2 Binding energy $(\Delta \mathrm{E})$ of the four complexes obtained by molecular dockings and binding energy $(\Delta \mathrm{G})$ obtained by MMPBSA method

\begin{tabular}{|c|c|c|c|c|}
\hline \multirow[t]{2}{*}{ Energy $(\mathrm{kJ} / \mathrm{mol})$} & \multicolumn{4}{|c|}{ Binding complex } \\
\hline & EACA & PAMBA & EDDA & AMCHA \\
\hline$\Delta \mathrm{E}_{\text {vdw_hb_desolv }}{ }^{\mathrm{c}}$ & -13.759 & $-12.42 \mid$ & -9.619 & -15.766 \\
\hline$\Delta \mathrm{E}_{\mathrm{ele}} \mathrm{c}^{\mathrm{c}}$ & -9.953 & $-|0.83|$ & -5.897 & -10.037 \\
\hline$\Delta \mathrm{E}_{\text {torsin }}{ }^{\mathrm{c}}$ & 8.740 & 4.977 & 11.208 & 4.977 \\
\hline$\Delta \mathrm{E}_{\text {bind }}{ }^{\mathrm{c}}$ & -14.972 & -18.275 & -4.307 & -20.785 \\
\hline$\Delta G_{v d w}$ & $\begin{array}{l}-31.600^{\mathrm{a}} \\
-36.0 \pm 0.1^{\mathrm{b}}\end{array}$ & $\begin{array}{l}-93.166^{\mathrm{a}} \\
-99.9 \pm 0.1^{\mathrm{b}}\end{array}$ & $\begin{array}{l}-72.186^{\mathrm{a}} \\
-67.4 \pm 0.1^{b}\end{array}$ & $\begin{array}{l}-60.982^{\mathrm{a}} \\
-69.0 \pm 0.2^{\mathrm{b}}\end{array}$ \\
\hline$\Delta \mathrm{G}_{\text {ele }}$ & $\begin{array}{l}-4.663^{\mathrm{a}} \\
-11.9 \pm 0.1^{\mathrm{b}}\end{array}$ & $\begin{array}{l}-3.516^{\mathrm{a}} \\
-14.5 \pm 0.1^{b}\end{array}$ & $\begin{array}{l}-12.648^{a} \\
-41.3 \pm 0.2^{b}\end{array}$ & $\begin{array}{l}3.912^{\mathrm{a}} \\
-4.7 \pm 0.2^{\mathrm{b}}\end{array}$ \\
\hline$\Delta \mathrm{G}_{\text {polar }}$ & $\begin{array}{l}17.364^{\mathrm{a}} \\
32.5 \pm 0.1^{\mathrm{b}}\end{array}$ & $\begin{array}{l}38.292^{\mathrm{a}} \\
42.2 \pm 0.1^{\mathrm{b}}\end{array}$ & $\begin{array}{l}41.564^{\mathrm{a}} \\
60.2 \pm 0.1^{\mathrm{b}}\end{array}$ & $\begin{array}{l}13.463^{\mathrm{a}} \\
32.4 \pm 0.1^{\mathrm{b}}\end{array}$ \\
\hline$\Delta \mathrm{G}_{\text {non-polar }}$ & $\begin{array}{l}-5.619^{a} \\
-5.6 \pm 0.0^{b}\end{array}$ & $\begin{array}{l}-9.056^{\mathrm{a}} \\
-8.6 \pm 0.0^{\mathrm{b}}\end{array}$ & $\begin{array}{l}-9.503^{a} \\
-8.8 \pm 0.0^{b}\end{array}$ & $\begin{array}{l}-8.277^{a} \\
-9.1 \pm 0.0^{b}\end{array}$ \\
\hline$\Delta \mathrm{G}_{\text {bind }}$ & $\begin{array}{l}-24.517^{\mathrm{a}} \\
-21.0 \pm 0.1^{\mathrm{b}}\end{array}$ & $\begin{array}{l}-67.491^{\mathrm{a}} \\
-80.8 \pm 0.2^{\mathrm{b}}\end{array}$ & $\begin{array}{l}-52.774^{\mathrm{a}} \\
-57.3 \pm 0.2^{\mathrm{b}}\end{array}$ & $\begin{array}{l}-51.885^{\mathrm{a}} \\
-50.4 \pm 0.2^{\mathrm{b}}\end{array}$ \\
\hline
\end{tabular}

Notes: a Obtained by g_mmpbsa. ' ${ }^{\circ}$ Obtained by gmxpbsatool. ' ${ }^{\circ}$ bbtained by autodock. $\Delta \mathrm{E}_{\text {bind }}$ : binding energy; $\Delta \mathrm{E}_{\mathrm{vdw}}$ hb desolv sum of $\mathrm{Van}$ der $\mathrm{Waals}$ energy, hydrogen bond energy and desolvation energy; $\Delta \mathrm{E}_{\text {ele }}$ : electrostatic energy; $\Delta \mathrm{E}_{\text {torsional }}$ : torsional energy; $\Delta \mathrm{G}_{\text {bind }}$ : binding free energy; $\Delta \mathrm{G}_{\mathrm{vdw}}:$ Van der Waals free energy; $\Delta \mathrm{G}_{\mathrm{ele}}$ : electrostatic free energy; $\Delta \mathrm{G}_{\text {polar }}$ : polar solvation free energy; and $\Delta \mathrm{G}_{\text {non-polar }}$ : non-polar salvation energy. Unit: $\mathrm{kJ} / \mathrm{mol}$.

Abbreviations: AMCHA, tranexamic acid; EACA, 6-aminocaproic acid; EDDA, ethylenediaminediacetic acid; PAMBA, p-(aminomethyl) benzoic acid. 

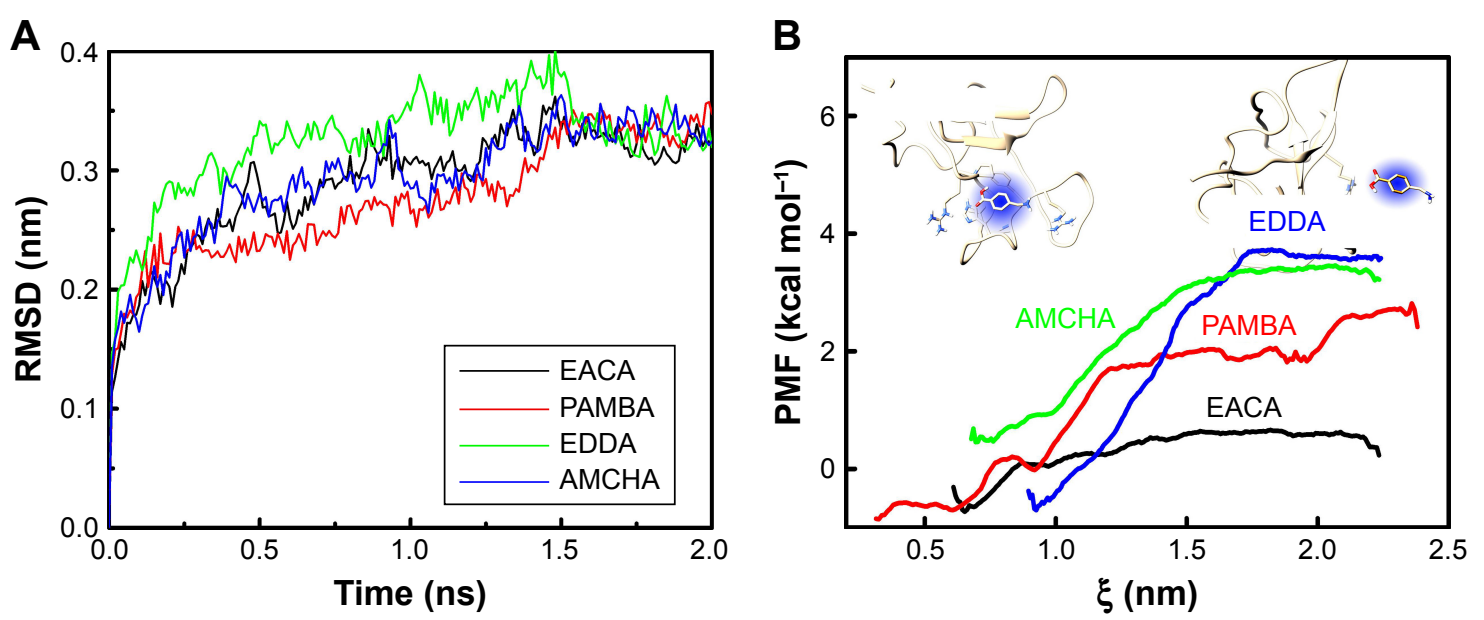

Figure 7 Results of MD simulations.

Notes: (A) RMSD of the backbone atoms of IHPK in the four docking complexes; (B) results of steered molecular dynamics obtained from umbrella sampling; inset was the typical result of PAMBA.

Abbreviations: AMCHA, tranexamic acid; EACA, 6-aminocaproic acid; EDDA, ethylenediaminediacetic acid; MD, molecular dynamics; PAMBA, p-(aminomethyl) benzoic acid; PMF, potential of mean force; RMSD, root mean square distance.

fitted well with the corresponding pocket (left inset in Figure 7B) because the carboxyl group of PAMBA was embedded deeply inside. The PMF curve of EDDA was very steep and arrived quickly at the topside from a $\xi$ of $1.0 \mathrm{~nm}$ to $1.7 \mathrm{~nm}$. For EACA and AMCHA, the $\xi$ did not increase in a short time. These results suggested that the PAMBA/1HPK complex was the most stable one among the four systems tested.

\section{Binding free energy}

To evaluate, quantitatively, the binding stability of the four drugs using the ligand with $1 \mathrm{HPK}$ as the target in the equilibrium complexes, the binding free energy $\left(\Delta G_{\text {bind }}\right)$ from the trajectory of MD was investigated further by MMPBSA (Table 2). $\Delta \mathrm{G}_{\text {bind }}$ was divided into four energies: Van der Waals energy $\left(\Delta \mathrm{G}_{\mathrm{vdw}}\right)$, electrostatic energy $\left(\Delta \mathrm{G}_{\text {ele }}\right)$, polar solvation energy $\left(\Delta \mathrm{G}_{\text {polar }}\right)$, and non-polar salvation energy $\left(\Delta \mathrm{G}_{\text {non-polar }}\right)$. The $\Delta \mathrm{G}_{\text {bind }}$ obtained for the four binding complexes was negative, indicating that binding was a spontaneous thermodynamic process. Like the results of the molecular-docking experiments, $\Delta \mathrm{G}_{\mathrm{vdw}}, \Delta \mathrm{G}_{\mathrm{ele}}$, and $\Delta \mathrm{G}_{\text {non-polar }}$ made favorable contributions to $\Delta \mathrm{G}_{\text {bind }}$, whereas $\Delta \mathrm{G}_{\text {polar }}$ made unfavorable contributions. $\Delta \mathrm{G}_{\mathrm{vdw}}$ was the main contributor among these three favorable subitems. $\Delta \mathrm{G}_{\text {bind }}$ was distinctly different from $\Delta \mathrm{E}_{\text {bind }}$ because: 1) the equilibrated conformation of the MD simulation had already changed considerably compared with the conformation of docking; 2) $\Delta G_{\text {bind }}$ was calculated from the sampling average of the trajectory whereas $\Delta \mathrm{E}_{\text {bind }}$ was obtained from the highest-score conformation. Moreover, MM-PBSA does not include an entropy calculation compared with autodock. The complex formed by PAMBA with $1 \mathrm{HPK}$ had the maximum $\Delta \mathrm{G}_{\text {bind }}$ $\left(-67.491 \mathrm{~kJ} \cdot \mathrm{mol}^{-1}\right)$, which indicated that the binding ability of the quasi-photo-stable PAMBA to $1 \mathrm{HPK}$ was the strongest. A possible reason for this result was the strong hydrophobic interaction of PAMBA with $1 \mathrm{HPK}$ protein resulting from the aromatic rings in PAMBA. The complex for EDDA had the largest $\Delta \mathrm{G}_{\text {ele }}\left(-12.648 \mathrm{~kJ} \cdot \mathrm{mol}^{-1}\right)$ and a larger $\Delta \mathrm{G}_{\mathrm{vdw}}$ $\left(-72.186 \mathrm{~kJ} \cdot \mathrm{mol}^{-1}\right)$, which led to enhanced binding behavior after reaching equilibrium. Interestingly, EACA reduced its binding affinity because of its small molecular volume, as evidenced by its lowest $\Delta \mathrm{G}_{\text {bind }}$. The energy model of MM-PBSA is biased toward larger ligands, awarding them higher scores (ie, more negative values) in comparison with smaller ligands. ${ }^{42}$ Hence, the energy score of EACA was affected by the target-dependence of the scoring function to some extent. Furthermore, under an identical condition, no restraint was applied to the binding between EACA and 1HPK even though EACA has more degrees of freedom and is accessed by bulk water. The larger $\Delta \mathrm{G}_{\text {ele }}$ was correlated closely to the hydrogen bond energy, suggesting that hydrogen bonds were quite important in the binding of EDDA to $1 \mathrm{HPK}$ protein but less important in the binding of EACA and PAMBA to 1 HPK protein (this will be discussed in detail in the following section describing interactions between drugs and 1HPK). According to Table 2, the results of gmxpbsatool were analogous to the results of g_mmpbsa, and provided the same trend: EACA $<$ AMCHA $<$ EDDA $<$ PAMBA.

To ascertain the contribution of amino-acid residues to $\Delta \mathrm{G}_{\text {bind }}$, the energy value for each contributing residue was obtained by MM-PBSA (Figure 8). The average contribution 


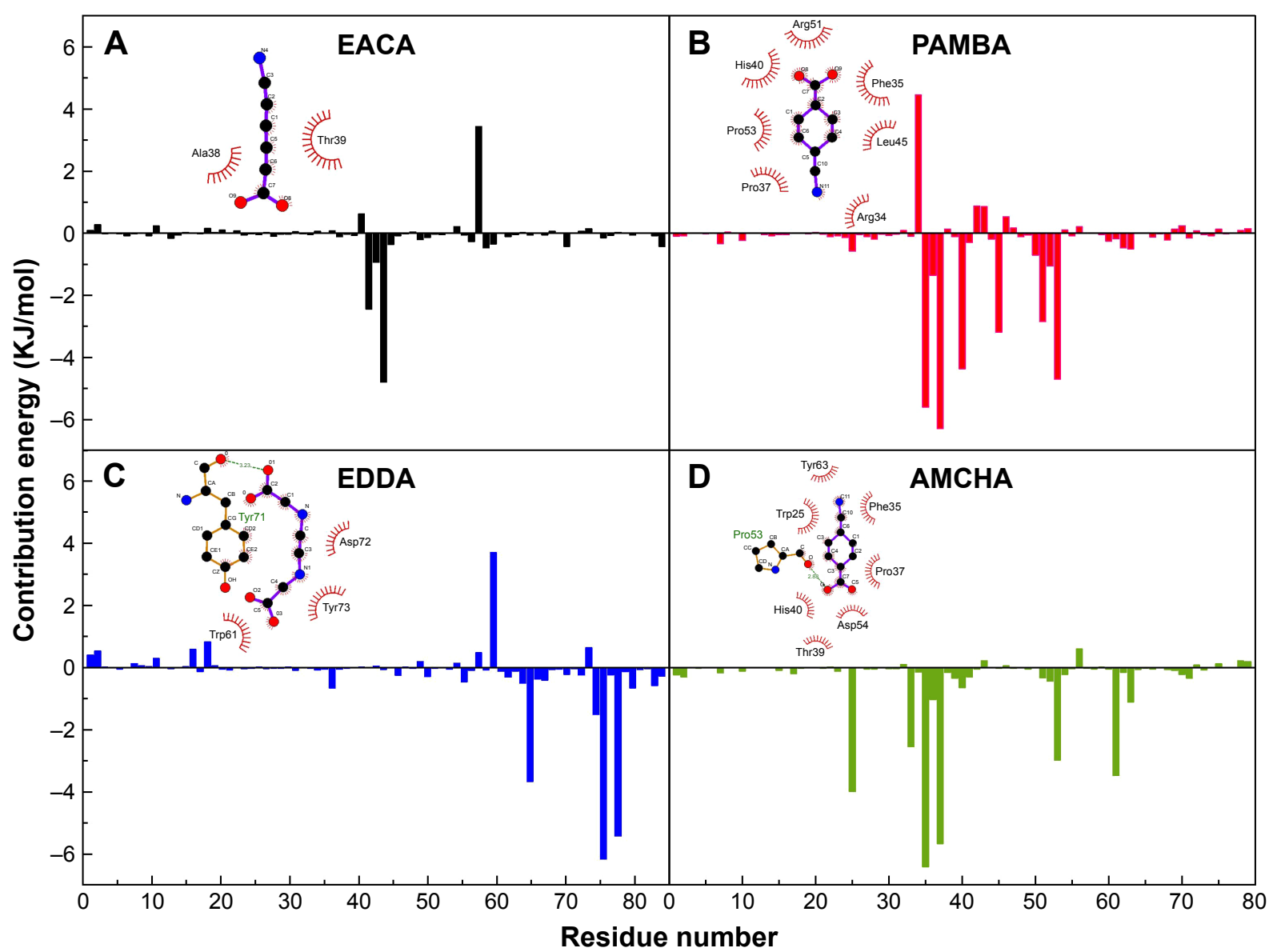

Figure 8 Interaction of MD simulations of equilibrium complexes and the corresponding contributions of individual amino acid residues in IHPK to the total binding energy. Note: (A) EACA, (B) PAMBA, (C) EDDA, and (D) AMCHA.

Abbreviations: AMCHA, tranexamic acid; EACA, 6-aminocaproic acid; EDDA, ethylenediaminediacetic acid; MD, molecular dynamics; PAMBA, p-(aminomethyl) benzoic acid.

of each amino acid residue to $\Delta \mathrm{G}_{\text {bind }}$ was quite different because of the difference in molecular conformations in the four types of complexes, even though $\Delta \mathrm{G}_{\text {bind }}$ was composed of the same residues. The contribution of each residue was described by numerical values (positive or negative), which could provide direct understanding of the composition of the binding energy. Results were also visualized by LigPlot and shown as insert maps at the top left of Figure 8. Here, we combined these two approaches to discuss this problem. Hydrophobic pockets were formed only by the surrounding two residues, so the binding affinity of EACA to 1HPK was the weakest among the four complexes. The residues in the hydrophobic pocket were negative, resulting in a maximum of $\Delta \mathrm{G}_{\text {bind }}$ (Figure $8 \mathrm{~B}$ ). Two proline molecules and one phenylalanine molecule had the strongest interaction with PAMBA. One hydrogen bond of length $3.23 \AA$ was between the $\mathrm{O}$ atom of Tyr71 and $\mathrm{H}$ atom of carboxyl in EDDA, which led to a maximum $\left(-6.1627 \mathrm{~kJ} \cdot \mathrm{mol}^{-1}\right)$ of Tyr71 (Figure 8C). The complex of AMCHA/1HPK shown in Figure 8D is used as a sample.
It shows that the drug interacted with 1HPK through hydrophobic means, and formed hydrophobic pockets with surrounding Trp25 $\left(-2.8501 \mathrm{~kJ} \cdot \mathrm{mol}^{-1}\right)$, Phe35 $\left(-4.5783 \mathrm{~kJ} \cdot \mathrm{mol}^{-1}\right)$, Pro37 $\left(-4.0464 \mathrm{~kJ} \cdot \mathrm{mol}^{-1}\right)$, Pro53 $\left(-2.1253 \mathrm{~kJ} \cdot \mathrm{mol}^{-1}\right)$, and other residues, including Thr39, His40, Asp54, and Tyr63, but these contributions were negligible. One hydrogen bond of length $2.88 \AA$ was also found between the $\mathrm{O}$ atom of Pro53 and $\mathrm{H}$ atom of a carboxyl group in AMCHA. However, this hydrogen bond could not make a huge contribution to $\Delta \mathrm{G}_{\text {bind }}$ because $\Delta \mathrm{G}_{\text {ele }}$ was found to be positive. According to the energy resolution, the hydrophobic interaction was shown to play an important part in the four types of complexes. Nevertheless, the hydrogen bond continued to play a key part in the binding of EDDA or AMCHA with 1HPK protein.

\section{Weak interactions between ligands and proteins}

To describe ligand-protein interactions, IGM was used to visualize the weak interaction between a hemostatic drug 
A
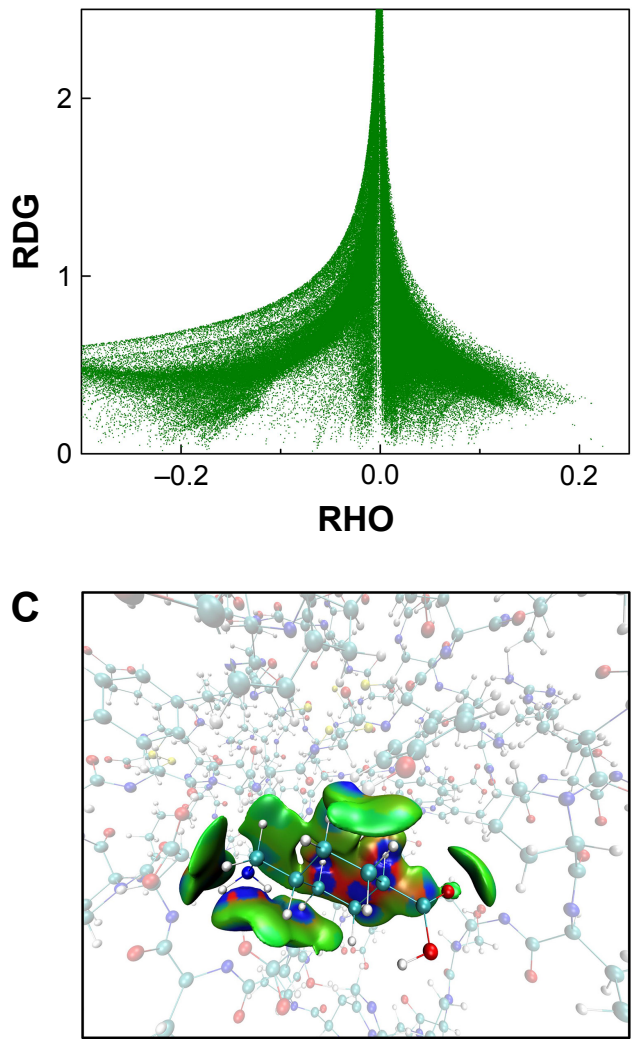

B

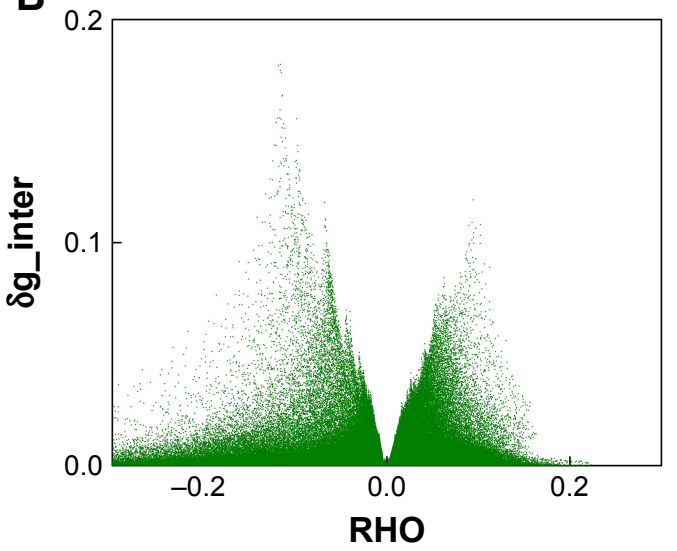

D

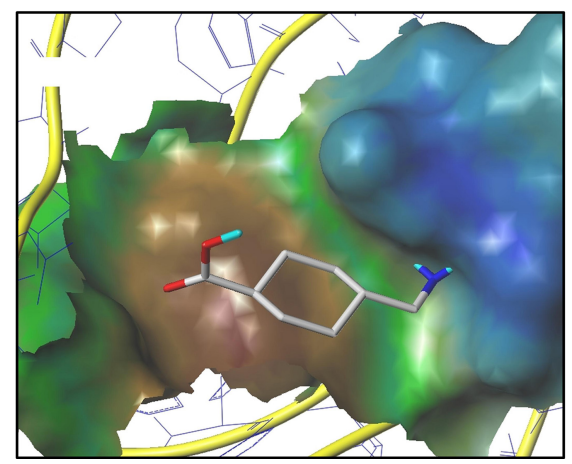

Figure 9 IGM weak interaction analysis of the complex of AMCHA/IHPK.

Note: (A) "Heel" value (RDG); (B) $\delta$ g_inter vs electron density (RHO); (C) Isosurface between AMCHA and IHPK, and (D) topology structure in the pocket of IHPK. Abbreviations: AMCHA, tranexamic acid; IGM, independent gradient model; RDG, reduced density gradient function.

and 1HPK (Figure 9). Typically, a weak interaction of AMCHA with 1 HPK protein was produced. The vertical bar in Figure 9A was called a "spike" on the leftmost side close to 0 , and this indicated a very weak interaction. All four complex systems had spikes on the left. Figure 9B shows an $\delta$ g_inter interaction, which was a strong hydrogen bond described as a peak along with Van der Waals forces. RHO is electron density, it is the main ingredient in such topological approach. This hydrogen bond was found within AMCHA because a peak occurred between RHO of 0.0 and RHO of -0.1 . Figure $9 \mathrm{C}$ depicts $3 \mathrm{D}$ spatial visualization by showing the iso-surfaces of AMCHA with 1HPK. The green isosurfaces for weak repulsive/attractive interactions (Van der Waals) appeared very large, and blue iso-surfaces for strong attractive interactions were located near the carboxylic-acid group. Figure 9D shows the electrostatic distribution in the pocket, which indicated that AMCHA was embedded deep in the target pocket.

\section{Discussion}

UV irradiation of fresh plasma does not alter its clotting mechanism. ${ }^{43,44}$ PSAE can elicit a hemostasis effect intraoperatively. However, repeated postpartum hemorrhage and uterine bleeding need to be present to employ the hemostatic drug in SAE to obtain long-term treatment effects. SAE has been shown to be a very good drug carrier based on its low viscosity and polymerizable property. Under microscopic observation during MIS, using a hemostatic drug has a better adhesive effect than spraying a hemostatic drug (eg, thrombin).

Under irradiation, the photo-stability of a drug is one of the most important parameters if the drug is to be used in MIS. TD-DFT as a potential screening method is a very useful tool to ascertain the photo-stability of a drug. This approach has been validated using UV photolysis. The photo-stability of a compound has a huge $\mathrm{E}_{\text {reorg }}$ because the PES of the excited state is far away from that of the ground state. It is caused by two huge $\mathrm{E}_{\text {relex }}$ values, which means that the vertical component for the excitation energy (Y-axis) and horizontal component for the translation (X-axis) are extremely large.

PAMBA could not bear UV irradiation according to the results of UV photolysis, even though it showed the strongest binding with $1 \mathrm{HPK}$ in simulations. In contrast, EACA showed the weakest binding with the protein among the four 
drugs tested, so EACA did not have sufficient affinity to exert hemostasis. EDDA showed photo-stability and satisfactory affinity, but had a slower release rate than that of AMCHA (as shown in drug-release experiments). The photo-stable AMCHA also had the highest $\Delta \mathrm{E}_{\text {bind }}\left(-20.785 \mathrm{~kJ} \cdot \mathrm{mol}^{-1}\right)$ and satisfactory $\Delta \mathrm{G}_{\text {bind }}\left(-51.885 \mathrm{~kJ} \cdot \mathrm{mol}^{-1}\right)$ according to simulation data. However, the membrane containing AMCHA was slightly opaque, and might affect intraoperative observations. This feature may be improved in the next scheme of drug modification. Overall, AMCHA was recommended to be the most suitable candidate used in MIS.

$\Delta \mathrm{G}$ from IGM represents electron density contragradience. We also obtained the sum of numerical value from $\mathrm{RHO}=0$ to $\mathrm{RHO}=-\infty, \mathrm{S}_{\text {igm }}^{\text {sg_inter }}$. They are 102 for EACA, 480 for PAMBA, 347 for EDDA, and 440 for AMCHA,

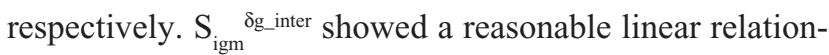
ship with $\Delta \mathrm{G}_{\text {bind }}$ (from mmpbsa) and $\Delta \mathrm{E}_{\text {bind }}$ (from docking), revealing that $\mathrm{S}_{\text {igm }}$ gg_inter could be treated as a candidate index to describe the binding energy between a ligand and a target. Using $\Delta \mathrm{G}$ as a descriptor of interaction energy can be very tempting. However, as emphasized by inventors, many questions remain, eg, $\Delta \mathrm{G}$ cannot be linked directly to interaction energy involving additional electrostatic contributions. More evidence is needed to find a relationship between the interaction energy and $\Delta \mathrm{G}$.

\section{Conclusion}

An integrated method bearing simultaneously the advantage of MIS and good adhesion capacity resulting from a sugarbased polymer system via photopolymerization was obtained to show better hemostasis efficiency in MIS. Except for the drug in PAMBA, the drug in EACA, AMCHA, and EDDA showed good photo-stability during photopolymerization according to a combination of spectroscopy measurements and quantum chemistry. Photopolymerization proceeded very rapidly and met the requirements for hemostasis in MIS. The formed film as a drug carrier could release the hemostatic drug for a long time, as confirmed by sustained-release experiments. Animal experiments in vivo showed that the film could achieve short-term, effective cure. Cytotoxicity experiments showed that the crosslinked matrix film had good bio-safety. Molecular-docking results revealed the docking sites of the four drugs binding to the targeted protein of $1 \mathrm{HPK}$, and also indicated that the conformation of $1 \mathrm{HPK}$ in each docking complex had changed greatly. Furthermore, simulations of binding free energy showed that AMCHA had better overall performance of the four drugs tested. Results obtained by experiments and molecular simulations showed that the carriers could pass through a trocar, bind to the tissue surface under photopolymerization, and then release AMCHA to enter the affected part over a long period of time.

\section{Acknowledgments}

This research was funded by the National Natural Science Foundation of China (21274032 and 51403043) and Natural Science Foundation of Guangdong Province (2014A030313500). It was also funded by a project of the high-level University of Guangdong Province and municipal college research program of the Guangzhou Education Bureau (1201610202). The general director and corresponding author, Guodong Ye, gratefully acknowledges the support for this study by the organizations mentioned previously. We also express our sincere gratitude to Professor Eric Hénon for detailed help using IGMPlot. Hong Huang, Houhe Liu, and Hua Zhou are co-first authors.

\section{Disclosure}

The authors report no conflicts of interest in this work.

\section{References}

1. Amanda MT, Gary NF. Cesarean scar defects: an underrecognized cause of abnormal uterine bleeding and other gynecologic complications. J Minim Invas Gyn. 2013;20(5):562-572.

2. de La Torre RA, Bachman SL, Wheeler AA, Bartow KN, Scott JS Hemostasis and hemostatic agents in minimally invasive surgery. Surgery. 2007;142(4 Supp1):S39-S45.

3. Mannucci PM. Hemostatic drugs. N Engl J Med. 1998;339(4):245-253.

4. Walter HS. Blood clotting mechanisms: three basic reactions. Ann Rev Physiol. 1969;31:269-294.

5. Storch AS, Rocha HNM, Garcia VP, et al. Oscillatory shear stress induces hemostatic imbalance in healthy men. Thromb Res. 2018;170(170) $119-125$.

6. Andrzejewska E. Photopolymerization kinetics of multifunctional monomers. Prog Polym Sci. 2001;26(4):605-665.

7. Nielsen VG, Ford PM. The ratio of concentrations of aminocaproic acid and tranexamic acid that prevent plasmin activation of platelets does not provide equivalent inhibition of plasmatic fibrinolysis. $J$ Thromb Thrombolysis. 2018;20.

8. Slaughter TF, Greenberg CS, Thomas FS, Charles SG. Antifibrinolytic drugs and perioperative hemostasis. Am J Hematol. 1997;56(1):32-36.

9. Wang GZ, Zhang CM, Sun LN. Influence of bleeding in endoscopic sinus surgery use of ethylenedia mine diaceturate. J Pract Med Tech. 2005;12(8):2222-2223.

10. Dunn CJ, Goa KL. Tranexamic acid. Drugs. 1999;57(6):1005-1032.

11. Saravana KJ, Arunpandian B, Muthu VV. Radiation-induced surface modification of polymers for biomaterial application. J Mater Sci. 2015;50(5):2007-2018.

12. Fisher JP, Dean D, Engel PS, Mikos AG. Photoinitiated polymerization of biomaterials. Annu Rev Mater Res. 2001;31(1):171-181.

13. Nilsson IM. Clinical pharmacology of tranexamic acid. Scand J Gastroentero Suppl. 1987;137:22-25.

14. Rejante MR, Llinás M. Solution structure of the epsilon-aminohexanoic acid complex of human plasminogen kringle 1. Eur J Biochem. 1994; 221(3):939-949

15. Motta A, Laursen RA, Llinás M, Tulinsky A, Park CH. Complete assignment of the aromatic proton magnetic resonance spectrum of the kringle 1 domain from human plasminogen: the structure of the ligand-binding site. Biochemistry. 1987;26(13):3827-3836. 
16. Magnusson S, Sottrup JL, Petersen TE. Homologous "kringle" structures common to plasminogen and prothrombin. substrate specificity of enzymes activating prothrombin and plasminogen. Proteoly Physiol Regul. 1976:203-238.

17. Patthy L, Trexler M, Váli Z, Bányai L, Váradi A. Kringles: modules specialized for protein binding. FEBS Lett. 1984;171(1):131-136.

18. Morris GM, Limwilby M. Molecular docking. Method Mol Biol. 2008;443:365-382.

19. Rossignol P, Ho-Tin-Noé B, Vranckx R, et al. Protease nexin-1 inhibits plasminogen activation-induced apoptosis of adherent cells. $J$ Biol Chem. 2004;279(11):10346-10356.

20. Fu JJ, Liu CC. Tri-block polymer with interfacial layer formation ability and its use in maintaining supersaturated drug solution after dissolution of solid dispersions. Int J Nanomedicine. 2018;13:1611-1619.

21. Yang JB. Nanjing well chemical Co., Ltd., assignee. synthetic method of sucrose allyl ether. China patent CN 1. 2007;944:449A.

22. Zhang Y, Song D, Huang H, et al. Minimally invasive hemostatic materials: tackling a dilemma of fluidity and adhesion by photopolymerization in situ. Sci Rep. 2017;7(1):15250.

23. Lee TY, Roper TM, Jonsson ES, et al. The kinetics of vinyl acrylate photopolymerization. Polymer. 2003;44(10):2859-2865.

24. Frisch MJ, Trucks GW, Schlegel HB, Scuseria GE, Robb MA, et al. Gaussian, Inc., Wallingford CT 06492 USA, 2016.

25. Berman HM, Westbrook J, Feng Z, et al. The protein data bank. Nucleic Acids Res. 2000;28(1):235-242.

26. Morris GM, Huey R, Lindstrom W, et al. AutoDock4 and AutoDockTools4: automated docking with selective receptor flexibility. J Comput Chem. 2009;30(16):2785-2791.

27. Yi L, Wang Y, Lin G, et al. Synthesis of conformation switchable cationic polypeptides based on poly(S-propargyl-cysteine) for use as siRNA delivery. Int J Biol Macromol. 2017;101:758-767.

28. van der Spoel D, Lindahl E, Hess B, Groenhof G, Mark AE, Berendsen HJ. GROMACS: fast, flexible, and free.JComput Chem. 2005; 26(16):1701-1718.

29. Kaminski GA, Friesner RA, Tirado-Rives J, Jorgensen WL. Evaluation and reparametrization of the OPLS-AA force field for proteins via comparison with accurate quantum chemical calculations on peptides. J Phys Chem B. 2001;105(28):6474-6487.

30. Schüttelkopf AW, van Aalten DM. PRODRG: a tool for high-throughput crystallography of protein-ligand complexes. Acta Crystallogr D Biol Crystallogr. 2004;60(Pt 8):1355-1363.

31. Fogolari F, Brigo A, Molinari H. Protocol for MM/PBSA molecular dynamics simulations of proteins. Biophys J. 2003;85(1):159-166.
32. Kumari R, Kumar R; Open Source Drug Discovery Consortium, Lynn A.g_mmpbsa-A GROMACS tool for high-throughput MM-PBSA calculations. J Chem Inf Model. 2014;54(7):1951-1962.

33. Paissoni C, Spiliotopoulos D, Musco G, Spitaleri A. GMXPBSA 2.0: a GROMACS tool to perform MM/PBSA and computational alanine scanning. Comput Phys Commun. 2014;185(11):2920-2929.

34. Wallace AC, Laskowski RA, Thornton JM. LIGPLOT: a program to generate schematic diagrams of protein-ligand interactions. Protein Eng Des Sel. 1995;8(2):127-134.

35. Johnson ER, Keinan S, Mori-Sánchez P, Contreras-García J, Cohen AJ, Yang W. Revealing noncovalent interactions. J Am Chem Soc. 2010; 132(18):6498-6506.

36. Lefebvre C, Rubez G, Khartabil H, Boisson JC, Contreras-García J, Hénon E. Accurately extracting the signature of intermolecular interactions present in the NCI plot of the reduced density gradient versus electron density. Phys Chem Chem Phys. 2017;19(27):17928-17936.

37. Zhang Y, Huang H, Liang ZL. The microscopic progression in the free radical addition reaction: modeling, geometry, energy, and kinetics. J Mol Model. 2017;23(73):1-10.

38. Luo Y, Xu X, An X, Sun X, Wang S, Zhu D. Targeted inhibition of the miR-199a/214 cluster by CRISPR interference augments the tumor tropism of human induced pluripotent stem cell-derived neural stem cells under hypoxic condition. Stem Cells Int. 2016;2016(3598542):1-8.

39. Eick JD, Kostoryz EL, Rozzi SM, et al. In vitro biocompatibility of oxirane/polyol dental composites with promising physical properties. Dent Mater. 2002;18(5):413-421.

40. Muzzarelli RAA, Muzzarelli C. Chitosan chemistry: relevance to the biomedical sciences. Adv Polym Sci. 2005;186:151-209.

41. Kang XJ, Caparas CIN, Soh BS, Fan Y. Addressing challenges in the clinical applications associated with CRISPR/Cas9 technology and ethical questions to prevent its misuse. Protein Cell. 2017;8(11): 791-795.

42. Genheden S, Ryde U. The MM/PBSA and MM/GBSA methods to estimate ligand-binding affinities. Expert Opin Drug Discov. 2015; 10(5):449-461.

43. Sherman R, Kaplan MD, Irving A, Friedman MD. Effects of ultravioletray irradiation on clotting mechanism of plasma. JAMA. 1951; 147(3):229-232.

44. Xie YJ, Yuan HM, Wang MB. Copy number variations independently induce autism spectrum disorder. Biosci Rep. 2017;37(4):1-8. 


\section{Supplementary materials}

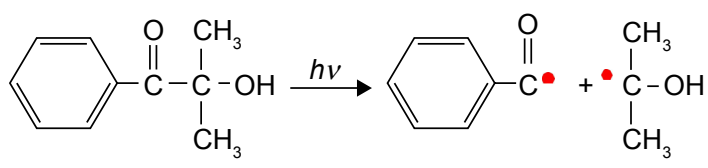

HMPP
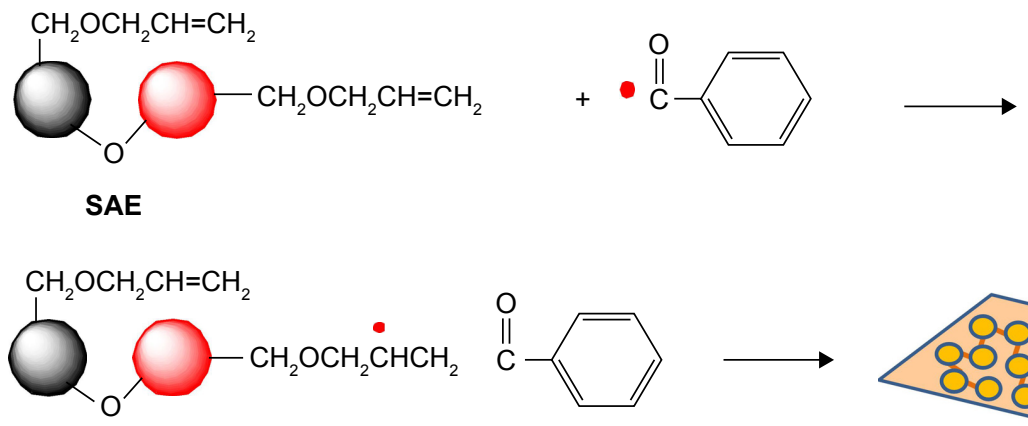<smiles>O=Cc1ccccc1</smiles><smiles>CCCCC</smiles>

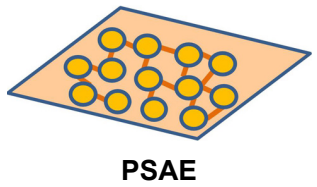<smiles>NCCCCCC(=O)O</smiles>

EACA<smiles>O=C(O)CNCCNCC(=O)O</smiles>

EDDA<smiles>NCc1ccc(C(=O)O)cc1</smiles>

PAMBA<smiles>NCC1CCC(C(=O)O)CC1</smiles>

AMCHA

Figure SI Photopolymerization reaction process of SAE with HMPP. The structure of four hemostatic drugs, AMCHA, EACA, EDDA and PAMBA.

Abbreviations: AMCHA, tranexamic acid; EACA, 6-aminocaproic acid; EDDA, ethylenediaminediacetic acid; HMPP, 2-hydroxy-2-methyl-I-phenyl-I-propanone; PAMBA, p-(aminomethyl) benzoic acid; PSAE, poly(sucrose allyl ether); SAE, sucrose allyl ether. 


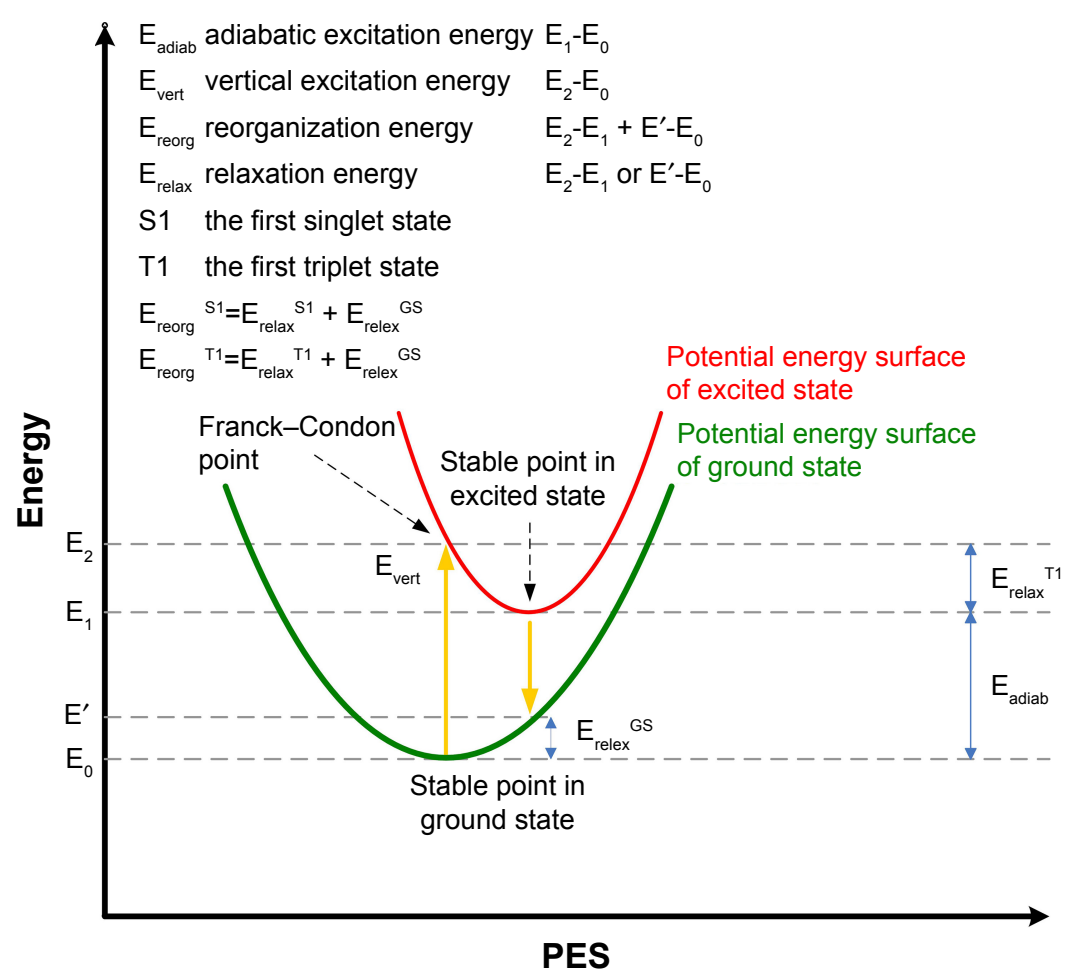

Figure S2 The illustration of excited energies.

Abbreviations: GS, ground state; PES, potential energy surface.

\section{Publish your work in this journal}

Drug Design, Development and Therapy is an international, peerreviewed open-access journal that spans the spectrum of drug design and development through to clinical applications. Clinical outcomes, patient safety, and programs for the development and effective, safe, and sustained use of medicines are the features of the journal, which has also been accepted for indexing on PubMed Central. The manuscript management system is completely online and includes a very quick and fair peer-review system, which is all easy to use. Visit http://www.dovepress.com/testimonials.php to read real quotes from published authors. 